\title{
FITOSSOCIOLOGIA DO COMPONENTE ARBÓREO DE UM FRAGMENTO DE FLORESTA OMBRÓFILA MISTA MONTANA, CURITIBA, PR, BR ${ }^{1}$
}

\author{
Carina Kozera*, Vinícius Antonio de Oliveira Dittrich**, Sandro Menezes Silva*** \\ *Bióloga, M.Sc., Doutoranda em Eng. Florestal, UFPR - kozera23@yahoo.com \\ **Biólogo, Dr., UNICSUL - vinarc@gmail.com \\ ***Biólogo, Dr., Conservação Internacional, CI Brasil - s.menezes@conservacao.org \\ Recebido para publicação: 18/06/2004 - Aceito para publicação: 10/06/2005
}

\begin{abstract}
Resumo
Realizou-se o estudo fitossociológico das espécies arbóreas da Floresta Ombrófila Mista Montana localizada no Parque Municipal do Barigüi, Curitiba, PR. Utilizando-se o método de quadrantes (pontos), foram alocados 150 pontos e amostrados indivíduos com perímetro do tronco à altura do peito (PAP) entre 10 e $29 \mathrm{~cm}$, para a caracterização das espécies de menor porte, e PAP igual ou superior a $30 \mathrm{~cm}$, para a caracterização das espécies de maior porte. No primeiro grupo foram registradas 77 espécies e 34 famílias, e no segundo, 67 espécies e 32 famílias. Em ambos os grupos, as famílias mais ricas em espécies foram Myrtaceae (17) e Lauraceae (oito). Encontrou-se maior diversidade no grupo de menor porte, resultante da associação das espécies com ocorrência exclusiva no estrato inferior e daquelas representadas por indivíduos em regeneração natural, representantes do grupo das espécies de maior porte que constituem o estrato superior da floresta.

Palavras-chave: Araucaria angustifolia; pinheiro-do-paraná; Floresta com Araucária; levantamento fitossociológico; Sul do Brasil.
\end{abstract}

\begin{abstract}
Phytosociology of the arboreal component of a patch of Mixed Ombrophilous Montane Forest at Curitiba, Paraná State, Brazil. A phytosociological study on the tree, species of the Mixed Ombrophilous Montane Forest at Barigüi Park, Municipality of Curitiba, Paraná State, Brazil, was carried out. The sampling method used was the point-centered quarter method. The criterion of inclusion adopted was: trees with PBH between 10 and $29 \mathrm{~cm}$ for the lower size stratum, and equal or above $30 \mathrm{~cm}$ for the higher size stratum. In total, 150 centered points were established. In the first group (the lower one) were found 77 species and 34 families, and in the second group 67 species and 32 families. In both groups, the richest families were Myrtaceae (17 species) and Lauraceae (eight). The highest diversity was found in the group with smaller size due to species' association of both strata (species exclusive from the lower stratum and species from the higher stratum represented by young individuals).

Keywords: Araucaria angustifolia; Brazilian pine; araucaria forest; phytosociological survey; Southern Brazil.
\end{abstract}

\section{INTRODUÇÃO}

A Floresta Ombrófila Mista (VELOSO et al. 1991), também conhecida como Floresta com Araucária ou Pinheiral, encontra-se distribuída no Brasil meridional, principalmente no planalto sulbrasileiro. Ocorre nos estados do Paraná, Santa Catarina e Rio Grande do Sul, na província de Misiones (Argentina), no sul do estado de São Paulo e na Serra da Mantiqueira (KLEIN, 1960). De acordo com IBGE (1992), pode ser subdividida nas formações Aluvial, Submontana, Montana e Altomontana, sendo essa classificação atribuída em função da latitude e da altitude de ocorrência da vegetação.

\footnotetext{
${ }^{1}$ Parte do projeto de pesquisa "Estudo florístico e fitossociológico da vegetação do Parque Barigüi, Curitiba, PR" (BANPESQ 95003945)
} 
As áreas ocupadas pela Floresta Ombrófila Mista (FOM) apresentam valores de precipitação média situados entre 1500 e $1750 \mathrm{~mm}$ anuais e temperatura variável. As médias térmicas no verão estão entre 20 e $21{ }^{\circ} \mathrm{C}$, e no inverno, entre 10 e $11^{\circ} \mathrm{C}$ (KLEIN, 1960).

A área de distribuição original da floresta cobria cerca de $175.000 \mathrm{~km}^{2}$. No entanto, por volta da década de 1990, já se encontrava reduzida a $20.000 \mathrm{~km}^{2}$ (LEITE; KLEIN, 1990). Entre os fatores responsáveis pela intensa redução da área de distribuição, estão a ocupação agrícola, o extrativismo vegetal e a instalação de empreendimentos agropecuários, associados à ausência de práticas adequadas e orientadas para o uso do solo.

Apesar de vários trabalhos sistematizados de levantamento florístico e/ou fitossociológico terem sido realizados no Brasil em áreas com FOM, ainda são necessários estudos dos remanescentes dessa unidade, principalmente com relação às espécies presentes na floresta e a forma como se distribuem na comunidade. Trabalhos dessa natureza contribuem para o conhecimento das características do ecossistema e podem subsidiar diferentes tipos de projetos, como por exemplo os de recuperação de áreas degradadas, auxiliando na seleção das espécies para a realização de plantios.

Na região Sul do Brasil, dentre os trabalhos com enfoque qualitativo e/ou quantitativo, realizados em FOM, citam-se o de Dombrowski e Kuniyoshi (1967), Klein (1963, 1979), Hatschbach e Moreira Filho (1972), Longhi (1980), Oliveira e Rotta (1980), Martau et al. (1981), Cestaro et al. (1986), Jarenkow e Baptista (1987), Cervi et al. (1987a, 1987b, 1988, 1989), Galvão et al. (1989), Roseira (1990), Silva e Marconi (1990), Machado et al. (1992), Negrelle e Silva (1992), Britez et al. (1995), Senna e Waechter (1997), Silva et al. (1997, 1998), Dittrich et al. (1999), Negrelle \& Leuchtenberger (2001) e Rondon Neto et al. (2002). Contribuíram, entre outros aspectos, para o conhecimento da composição e da estrutura de diferentes comunidades vegetacionais.

Com a mesma finalidade, realizou-se o levantamento fitossociológico de um fragmento de FOM situado em um parque municipal da região urbana do município de Curitiba. Foram objetivos do trabalho registrar as espécies constituintes do componente arbóreo e caracterizar a estrutura da floresta através do uso de parâmetros fitossociológicos usualmente utilizados como descritores da comunidade vegetacional. A área de estudo destaca-se em importância por representar um remanescente das florestas que cobriam grande parte do município, bem como área de abrigo e de recursos para a fauna nativa da região.

\section{MATERIAL E MÉTODOS}

O estudo fitossociológico foi realizado no fragmento de Floresta Ombrófila Mista Montana localizado no Parque Municipal do Barigüi, Curitiba, PR. A área está situada a 900 m s.n.m. e apresenta cerca de 17,5 ha (ABE et al. 2000).

O clima na região, segundo a classificação de Köppen, é do tipo Cfb, subtropical úmido, mesotérmico de verões frescos, inverno com geadas freqüentes, sem estação seca (IAPAR, 1978). A temperatura média anual é de $16,7{ }^{\circ} \mathrm{C}$, sendo a temperatura média mensal de $20,5^{\circ} \mathrm{C}$ no mês mais quente (fevereiro) e $13{ }^{\circ} \mathrm{C}$ nos meses mais frios (junho e julho). A precipitação média mensal varia de $168,9 \mathrm{~mm}$ em janeiro a 75,6 mm em agosto (ROSEIRA, 1990).

Para a realização do levantamento, foi utilizado o método de pontos ou quadrantes (MARTINS, 1993), sendo que em cada um dos quadrantes definidos no momento da amostragem foram registrados dois indivíduos arbóreos: um deles com perímetro do tronco à altura do peito (PAP) entre 10 e $29 \mathrm{~cm}$ e o outro com PAP igual ou superior a $30 \mathrm{~cm}$. Os pontos foram alocados em intervalos regulares de $10 \mathrm{~m}$.

Os dois intervalos de medidas de PAP foram definidos como critério de inclusão com o objetivo de caracterizar, supostamente, os indivíduos arbóreos da floresta com menor e maior porte, respectivamente. Partiu-se da premissa de que os indivíduos arbóreos com menor perímetro de tronco apresentariam menores valores de altura e estariam presentes na comunidade ocupando o estrato inferior, por isso denominados de menor porte. Já os indivíduos arbóreos com maior perímetro do tronco apresentariam maiores alturas e estariam presentes na floresta ocupando o estrato superior, por isso denominados de maior porte.

Em campo, foram anotados a altura, o PAP e a distância da base do tronco de cada indivíduo amostrado ao ponto da amostragem e coletado material botânico para posterior determinação. Os procedimentos de coleta e herborização utilizados seguiram as técnicas citadas em IBGE (1992). 
Para a determinação, foram utilizadas bibliografia específica para as famílias, gêneros e espécies, comparações com exsicatas dos Herbários UPCB (Departamento de Botânica da Universidade Federal do Paraná) e MBM (Museu Botânico Municipal de Curitiba), e consultas a especialistas em taxonomia vegetal. Para a conferência da escrita dos nomes científicos e a autoria dos epítetos específicos, foram consultados os bancos de dados eletrônicos do Jardim Botânico de Missouri (Missouri Botanical Garden, 2006) e do IPNI (IPNI - The International Plant Names Index, 2006). Os materiais coletados férteis foram depositados no Herbário UPCB e as duplicatas no MBM.

Todos os dados coletados foram tratados com o programa FITOPAC (SHEPHERD, 1995). Foram determinados os seguintes parâmetros fitossociológicos: freqüência, densidade, dominância, valor de importância, índice de diversidade de Shannon e equabilidade.

\section{RESULTADOS}

Foram alocados 150 pontos correspondentes ao levantamento de 1200 indivíduos pertencentes a 97 espécies e 40 famílias. Duas dessas pertencem a pteridófita (Cyatheaceae e Dicksoniaceae), duas a gimnosperma (Araucariaceae e Podocarpaceae) e as demais (36) a angiosperma (Tabelas 1 e 2).

Tabela 1. Valores obtidos no estudo fitossociológico da Floresta Ombrófila Mista Montana do Parque Municipal do Barigüi (Curitiba, PR) para as espécies com PAP entre 10 e $29 \mathrm{~cm}$ e com PAP igual ou maior do que $30 \mathrm{~cm}$.

Table 1. Obtained values in the phytosociological study of the Mixed Ombrophilous Montane Forest at Barigüi Park (Curitiba, Paraná) for the species with PBH between 10 and $29 \mathrm{~cm}$ (left), and species equal or above $30 \mathrm{~cm}$ of PBH (right).

\begin{tabular}{lll}
\hline Parâmetros & $\begin{array}{l}\text { PAP entre 10-29 cm } \\
\text { (grupo “menor porte") }\end{array}$ & $\begin{array}{l}\text { PAP } \geq \mathbf{3 0} \text { cm } \\
\text { (grupo “maior porte") }\end{array}$ \\
\hline Número de espécies & 77 & 67 \\
Número de famílias & 34 & 32 \\
Distância média (m) & 2,93 & 3,95 \\
Densidade total (indivíduos/ha) & 1166,70 & 641,56 \\
Área basal total (m ${ }^{2} /$ ha) & 1,410 & 33,252 \\
Índice de diversidade H'(nats/indivíduo) & 3,579 & 2,708 \\
Equabilidade & 0,824 & 0,644 \\
Área equivalente da amostra (ha) & 0,514 & 0,935 \\
Altura média (m) & 6,49 & 13,05 \\
Desvio padrão da altura (m) & 2,17 & 3,50 \\
\hline
\end{tabular}

As famílias mais ricas em espécies foram Myrtaceae (17), Lauraceae (oito), Aquifoliaceae (seis), Fabaceae (seis) e Flacourtiaceae (cinco).

A amostragem dos indivíduos arbóreos, em função dos dois intervalos de PAP, possibilitou a caracterização de dois grupos de espécies: o de menor (PAP entre 10 e $29 \mathrm{~cm}$ ) e o de maior porte (PAP igual ou superior a $30 \mathrm{~cm}$ ), assim denominados neste trabalho.

Os indivíduos amostrados como de menor porte apresentaram altura média igual a $6,5 \mathrm{~m}$. Encontram-se no interior do fragmento florestal representando o estrato inferior. Aqueles denominados de maior porte tiveram registro da altura média igual a $13 \mathrm{~m}$ e encontram-se, com exceção das samambaias arborescentes, no estrato superior.

No grupo definido como de menor porte, foram registradas 77 espécies, 52 gêneros e 34 famílias. Myrtaceae (16), Aquifoliaceae (cinco), Flacourtiaceae (cinco) e Lauraceae (cinco) foram as mais ricas em espécies e as mais abundantes, totalizando $65 \%$ do número total de árvores amostradas. Casearia decandra Jacq., Ocotea corymbosa (Meisn.) Mez, Ocotea nutans (Nees) Mez, Mosiera prismatica (Legrand) Landrum, Casearia sylvestris Sw., Casearia obliqua Spreng., Cordiera concolor (Cham.) O. Kuntze, Allophylus edulis (A. St.-Hil., Cambess. \& A. Juss.) Radlk., Myrceugenia miersiana (Gardner) Legrand e Kausel e Psychotria vellosiana Benth. destacaram-se com os maiores valores de importância (VI), totalizando $55 \%$ do valor total da amostra. Casearia decandra Jacq. apresentou maior valor de freqüência, densidade e dominância relativas (Tabela 3). 
Tabela 2. Espécies amostradas na Floresta Ombrófila Mista Montana do Parque Municipal do Barigüi (Curitiba, PR), com indicação da ocorrência no grupo das espécies com PAP entre 10 e $29 \mathrm{~cm}$ ("A") e/ou no grupo com PAP igual ou superior a $30 \mathrm{~cm}$ ("B"). UPCB = número de registro no herbário do Departamento de Botânica da UFPR; s/col. = sem coleta de material fértil.

Table 2. Sampled species at the Mixed Ombrophilous Montane Forest at Barigüi Park (Curitiba, Paraná) showing the occurrence in the lower size group (PBH between 10 and $29 \mathrm{~cm}$ - "A") and/or in the higher size group (PBH equal or above $30 \mathrm{~cm}$ - "B"). UPCB = register number at the Herbarium from the Botany Department of the Universidade Federal do Paraná, s/col. = not collected.

\begin{tabular}{|c|c|c|c|c|}
\hline Família / Espécie & Nome popular & $\mathbf{A}$ & B & UPCB \\
\hline \multicolumn{5}{|l|}{ ANACARDIACEAE } \\
\hline Lithraea brasiliensis Marchand & pau-de-bugre & $\mathrm{x}$ & $\mathrm{x}$ & 28384 \\
\hline Schinus terebinthifolius Raddi & aroeira-vermelha & & $\mathrm{x}$ & 25987 \\
\hline \multicolumn{5}{|l|}{ ANNONACEAE } \\
\hline Rollinia emarginata Schltdl. & araticum-do-mato & $\mathrm{x}$ & & 28581 \\
\hline \multicolumn{5}{|l|}{ AQUIFOLIACEAE } \\
\hline Ilex amara Loes. & caúna-miúda & $\mathrm{x}$ & & s/col. \\
\hline Ilex brevicuspis Reissek & caúna & $\mathrm{x}$ & $\mathrm{x}$ & $\mathrm{s} / \mathrm{col}$. \\
\hline Ilex dumosa Reissek & congonha-miúda & & $\mathrm{x}$ & 27098 \\
\hline Ilex paraguariensis A. St.-Hil. & erva-mate & $\mathrm{x}$ & & 28582 \\
\hline Ilex theezans Mart. & orelha-de-mico, caúna & $\mathrm{x}$ & $\mathrm{x}$ & $\mathrm{s} / \mathrm{col}$. \\
\hline Ilex sp. & - & $\mathrm{x}$ & & s/col. \\
\hline \multicolumn{5}{|l|}{ ARALIACEAE } \\
\hline Tetrapanax papyriferum K. Koch* & - & $\mathrm{x}$ & & $\mathrm{s} / \mathrm{col}$. \\
\hline \multicolumn{5}{|l|}{ ARAUCARIACEAE } \\
\hline Araucaria angustifolia (Bertol.) Kuntze & pinheiro-do-paraná & & $\mathrm{x}$ & 25981 \\
\hline \multicolumn{5}{|l|}{ ARECACEAE } \\
\hline Syagrus romanzoffiana (Cham.) Glassman & jerivá & & $\mathrm{x}$ & $\mathrm{s} / \mathrm{col}$. \\
\hline \multicolumn{5}{|l|}{ ASTERACEAE } \\
\hline Baccharis brachylaenoides DC. & vassoura & $\mathrm{x}$ & & 39649 \\
\hline Vernonia discolor (Spreng.) Less. & vassourão-preto & & $\mathrm{x}$ & 28315 \\
\hline \multicolumn{5}{|l|}{ BIGNONIACEAE } \\
\hline Jacaranda puberula Cham. & caroba & & $\mathrm{x}$ & 25637 \\
\hline \multicolumn{5}{|l|}{ CANELLACEAE } \\
\hline Capsicodendron dinisii (Schwacke) Occhioni & pimenteira & $\mathrm{x}$ & $\mathrm{x}$ & $\mathrm{s} / \mathrm{col}$. \\
\hline \multicolumn{5}{|l|}{ CELASTRACEAE } \\
\hline Maytenus alaternoides Reissek & coração-de-bugre & $\mathrm{x}$ & & $\mathrm{s} / \mathrm{col}$. \\
\hline Maytenus aquifolium Mart. & - & & $\mathrm{x}$ & $\mathrm{s} / \mathrm{col}$. \\
\hline Maytenus evonymoides Reissek & coração-de-bugre & & $\mathrm{x}$ & 26041 \\
\hline Maytenus ilicifolia (Schrad.) Planch. & espinheira-santa & $\mathrm{x}$ & & $\mathrm{s} / \mathrm{col}$. \\
\hline \multicolumn{5}{|l|}{ CLETHRACEAE } \\
\hline Clethra scabra Pers. & carne-de-vaca & $\mathrm{x}$ & $\mathrm{x}$ & $\mathrm{s} / \mathrm{col}$. \\
\hline \multicolumn{5}{|l|}{ CUNONIACEAE } \\
\hline Lamanonia ternata Vell. & guaperê & $\mathrm{x}$ & $\mathrm{x}$ & 29815 \\
\hline \multicolumn{5}{|l|}{ CYATHEACEAE } \\
\hline Alsophila setosa Kaulf. & samambaiuçu & $\mathrm{x}$ & $\mathrm{x}$ & 26031 \\
\hline \multicolumn{5}{|l|}{ DICKSONIACEAE } \\
\hline Dicksonia sellowiana Hook. & xaxim, xaxim-bugio & & $\mathrm{x}$ & $\mathrm{s} / \mathrm{col}$. \\
\hline \multicolumn{5}{|l|}{ ELAEOCARPACEAE } \\
\hline Sloanea monosperma Vell. & sapopema & $\mathrm{x}$ & & $\mathrm{s} / \mathrm{col}$. \\
\hline \multicolumn{5}{|l|}{ ERYTHROXILACEAE } \\
\hline Erythroxylum deciduum A. St.-Hil. & cocão, fruto-de-pomba & & $\mathrm{x}$ & 26042 \\
\hline \multicolumn{5}{|l|}{ EUPHORBIACEAE } \\
\hline Sebastiania brasiliensis Spreng. & leiteiro-branco & $\mathrm{x}$ & $\mathrm{x}$ & 26077 \\
\hline Sebastiania commersoniana (Baill.) L. B. Sm. \& Downs & branquilho & $\mathrm{x}$ & $\mathrm{x}$ & 25718 \\
\hline FABACEAE & & & & \\
\hline Dalbergia frutescens (Vell.) Britton & rabo-de-bugio & $\mathrm{x}$ & & 29329 \\
\hline Lonchocarpus cultratus (Vell.) A. M. G. Azevedo \& H. C. Lima & - & & $\mathrm{x}$ & $\mathrm{s} / \mathrm{col}$. \\
\hline
\end{tabular}




\begin{tabular}{|c|c|c|c|c|}
\hline Família / Espécie & Nome popular & $\mathbf{A}$ & $\mathbf{B}$ & UPCB \\
\hline Lonchocarpus subglaucescens Mart. ex Benth. & timbó & $\mathrm{x}$ & $\mathrm{x}$ & 27128 \\
\hline Machaerium brasiliense Vogel & sapuva & $\mathrm{x}$ & $\mathrm{x}$ & $\mathrm{s} / \mathrm{col}$. \\
\hline Machaerium paraguariense Hassl. & jacarandá-branco & $\mathrm{x}$ & & $\mathrm{s} / \mathrm{col}$. \\
\hline $\begin{array}{l}\text { Machaerium stipitatum (DC.) Vogel } \\
\text { FLACOURTIACEAE }\end{array}$ & sapuva & & $\mathrm{x}$ & $\mathrm{s} / \mathrm{col}$. \\
\hline Banara parviflora (A. Gray) Benth. & cabroé-mirim & $\mathrm{x}$ & & s/col. \\
\hline Casearia decandra Jacq. & guaçatunga & $\mathrm{x}$ & $\mathrm{x}$ & 28316 \\
\hline Casearia obliqua Spreng. & guaçatunga & $\mathrm{x}$ & $\mathrm{x}$ & 25997 \\
\hline Casearia sylvestris $\mathrm{Sw}$. & cafezeiro-do-mato & $\mathrm{x}$ & $\mathrm{x}$ & 25644 \\
\hline $\begin{array}{l}\text { Xylosma ciliatifolium } \text { Eichl. } \\
\text { LAURACEAE }\end{array}$ & sucará & $\mathrm{x}$ & $\mathrm{x}$ & 25719 \\
\hline Cinnamomum amoenum (Nees) Kosterm. & canela & $\mathrm{x}$ & $\mathrm{x}$ & 30580 \\
\hline Cinnamoтum sp. & - & $\mathrm{x}$ & & $\mathrm{s} / \mathrm{col}$ \\
\hline Cryptocarya aschersoniana $\mathrm{Mez}$ & canela-amarela & & $\mathrm{x}$ & 28558 \\
\hline Ocotea corymbosa (Meisn.) Mez & canela-preta & $\mathrm{x}$ & $\mathrm{x}$ & s/col. \\
\hline Ocotea nutans (Nees) Mez & canela & $\mathrm{x}$ & $\mathrm{x}$ & 27456 \\
\hline Ocotea puberula (Rich.) Nees & canela-guaicá & & $\mathrm{x}$ & s/col. \\
\hline Ocotea pulchella (Nees) Mez & canela-lageana & $\mathrm{x}$ & $\mathrm{x}$ & 27455 \\
\hline Persea major (Nees) L. E. Kopp & - & & $\mathrm{x}$ & 29343 \\
\hline LOGANIACEAE & & & & \\
\hline $\begin{array}{l}\text { Strychnos brasiliensis (Spreng.) Mart. } \\
\text { LYTHRACEAE }\end{array}$ & - & $\mathrm{x}$ & & 29345 \\
\hline $\begin{array}{l}\text { Lafoensia pacari A. St.-Hil. } \\
\text { MELIACEAE }\end{array}$ & dedaleiro & $\mathrm{x}$ & $\mathrm{x}$ & 29347 \\
\hline Cabralea canjerana (Vell.) Mart. & canjerana & $\mathrm{x}$ & $\mathrm{x}$ & 27136 \\
\hline $\begin{array}{l}\text { Cedrela } \text { cf. odorata } \mathrm{L} . \\
\text { MONIMIACEAE }\end{array}$ & cedro & $\mathrm{x}$ & & $\mathrm{s} / \mathrm{col}$ \\
\hline $\begin{array}{l}\text { Mollinedia clavigera Tul. } \\
\text { MYRSINACEAE }\end{array}$ & capixim & $\mathrm{x}$ & & 26086 \\
\hline Rapanea lancifolia $\mathrm{Mez}$ & capororoca & & $\mathrm{x}$ & s/col. \\
\hline $\begin{array}{l}\text { Rapanea umbellata (Mart.) Mez } \\
\text { MYRTACEAE }\end{array}$ & capororocão & $\mathrm{x}$ & $\mathrm{x}$ & 25747 \\
\hline Blepharocalyx salicifolius (Kunth) O. Berg & murta, guamirim & $\mathrm{x}$ & $\mathrm{x}$ & s/col. \\
\hline Calyptranthes concinna DC. & guamirim-facho & $\mathrm{x}$ & & 28327 \\
\hline Campomanesia xanthocarpa $\mathrm{O}$. Berg & guabirobeira-do-mato & $\mathrm{x}$ & $\mathrm{x}$ & 25748 \\
\hline Eugenia platysema $\mathrm{O}$. Berg & - & $\mathrm{x}$ & & 30655 \\
\hline Eugenia uniflora $\mathrm{L}$ & pitanga & $\mathrm{x}$ & $\mathrm{x}$ & 27139 \\
\hline Mosiera prismatica (D. Legrand) Landrum & cerninho, cambuí & $\mathrm{x}$ & $\mathrm{x}$ & 27138 \\
\hline Myrceugenia myrcioides (Cambess.) O. Berg & guamirim & $\mathrm{x}$ & $\mathrm{x}$ & 39627 \\
\hline Myrceugenia miersiana (Gardner) D. Legrand \& Kausel & guamirim-da-várzea & $\mathrm{x}$ & $\mathrm{x}$ & 27141 \\
\hline Myrceugenia ovata (Hook. \& Arn.) O. Berg & - & $\mathrm{x}$ & & 27144 \\
\hline Myrcia hatschbachii D. Legrand & caingá & $\mathrm{x}$ & $\mathrm{x}$ & 29357 \\
\hline Myrcia multiflora (Lam.) DC. & cambuí & $\mathrm{x}$ & $\mathrm{x}$ & 29358 \\
\hline Myrcia obtecta (O. Berg) Kiaersk. & cambuí, guamirim-branco & $\mathrm{x}$ & $\mathrm{x}$ & 28559 \\
\hline Myrcia rostrata DC. & guamirim-de-folha-miúda & $\mathrm{x}$ & $\mathrm{x}$ & $\mathrm{s} / \mathrm{col}$ \\
\hline Myrcia cf. venulosa DC. & guamirim & $\mathrm{x}$ & & 39646 \\
\hline Myrcia sp. & - & & $\mathrm{x}$ & s/col. \\
\hline Myrciaria tenella (DC.) O. Berg & cambuí & $\mathrm{x}$ & & 30122 \\
\hline $\begin{array}{l}\text { Pimenta pseudocaryophyllus (Gomes) Landrum } \\
\text { PODOCARPACEAE }\end{array}$ & craveiro-do-mato & $\mathrm{x}$ & $\mathrm{x}$ & s/col. \\
\hline $\begin{array}{l}\text { Podocarpus lambertii Klotzsch ex Endl. } \\
\text { PROTEACEAE }\end{array}$ & pinheiro-bravo & $\mathrm{x}$ & $\mathrm{x}$ & 25982 \\
\hline $\begin{array}{l}\text { Roupala brasiliensis Klotzsch } \\
\text { RHAMNACEAE }\end{array}$ & carvalho-brasileiro & $\mathrm{x}$ & & $\mathrm{s} / \mathrm{col}$. \\
\hline $\begin{array}{l}\text { Hovenia dulcis Thunb.* } \\
\text { ROSACEAE }\end{array}$ & uva-do-japão & $\mathrm{x}$ & & 29374 \\
\hline Prunus sellowii Koehne & pessegueiro-bravo & $\mathrm{x}$ & $\mathrm{x}$ & 26059 \\
\hline
\end{tabular}

FLORESTA, Curitiba, PR, v. 36, n. 2, mai./ago. 2006. 


\begin{tabular}{|c|c|c|c|c|}
\hline Família / Espécie & Nome popular & $\mathbf{A}$ & B & UPCB \\
\hline \multicolumn{5}{|l|}{ RUBIACEAE } \\
\hline Cordiera concolor (Cham.) O. Kuntze & - & $\mathrm{x}$ & & 27147 \\
\hline Guettarda uruguensis Cham. \& Schltdl. & veludinho & $\mathrm{x}$ & & 29376 \\
\hline Psychotria vellosiana Benth. & - & $\mathrm{x}$ & $\mathrm{x}$ & 28338 \\
\hline Psychotria suterella Muell. Arg. & _ & $\mathrm{x}$ & & 27416 \\
\hline \multicolumn{5}{|l|}{ RUTACEAE } \\
\hline Zanthoxylum kleinii (R.S. Cowan) P.G. Waterman & juvevê & & $\mathrm{x}$ & $\mathrm{s} / \mathrm{col}$ \\
\hline $\begin{array}{l}\text { Zanthoxylum rhoifolium Lam. } \\
\text { SAPINDACEAE }\end{array}$ & \multicolumn{3}{|c|}{ SAPINDACEAE } & 25754 \\
\hline Allophylus edulis (A. St.-Hil., Cambess. \& A. Juss.) Radlk. & vacum & $\mathrm{x}$ & $\mathrm{x}$ & 25658 \\
\hline Allophylus guaraniticus (A. St.-Hil.) Radlk. & vacum & $\mathrm{x}$ & $\mathrm{x}$ & 25660 \\
\hline Cupania vernalis Cambess. & cuvantã & $\mathrm{x}$ & & $\mathrm{s} / \mathrm{col}$ \\
\hline $\begin{array}{l}\text { Matayba elaeagnoides Radlk. } \\
\text { SIMAROUBACEAE }\end{array}$ & miguel-pintado & $\mathrm{x}$ & $\mathrm{x}$ & 28563 \\
\hline $\begin{array}{l}\text { Picramnia parvifolia } \text { Engl. } \\
\text { SOLANACEAE }\end{array}$ & pau-amargo & $\mathrm{x}$ & & 28584 \\
\hline Solanum granuloso-leprosum Dunal & - & & $\mathrm{x}$ & 28046 \\
\hline Solanum pseudoquina A. St.-Hil. & joá-de-árvore & $\mathrm{x}$ & $\mathrm{x}$ & 26114 \\
\hline Solanum sanctaecatharinae Dunal & joá, joá-manso & $\mathrm{x}$ & $\mathrm{x}$ & 28586 \\
\hline \multicolumn{5}{|l|}{ STYRACACEAE } \\
\hline \multicolumn{5}{|l|}{ SYMPLOCACEAE } \\
\hline Symplocos $\mathrm{sp}$. & - & $\mathrm{x}$ & & $\mathrm{s} / \mathrm{col}$ \\
\hline $\begin{array}{l}\text { Symplocos tetrandra Mart. } \\
\text { ULMACEAE }\end{array}$ & - & $\mathrm{x}$ & $\mathrm{x}$ & 27425 \\
\hline $\begin{array}{l}\text { Celtis tala Gillies ex Planch. } \\
\text { VERBENACEAE }\end{array}$ & - & $\mathrm{x}$ & $\mathrm{x}$ & 29387 \\
\hline Duranta vestita Cham. & baga-de-pomba & $\mathrm{x}$ & $\mathrm{x}$ & 25766 \\
\hline Lantana brasiliensis Link & - & $\mathrm{x}$ & & 26125 \\
\hline WINTERACEAE & & & & \\
\hline Drimys brasiliensis Miers & cataia & $\mathrm{x}$ & $\mathrm{x}$ & 26007 \\
\hline
\end{tabular}

* Espécie exótica, de acordo com Graf(1992) e/ou Lorenzi et al. (2003)

O grupo de espécies de maior porte foi representado por 67 espécies, 45 gêneros e 32 famílias. Podocarpaceae destacou-se com o maior número de indivíduos, cerca de $43 \%$ do total de árvores amostradas. Nesse grupo, Myrtaceae (12) e Lauraceae (sete) foram as que apresentaram o maior número de espécies. Podocarpus lambertii Klotzsch, Mosiera prismatica (Legrand) Landrum, Araucaria angustifolia (Bertol.) Kuntze e Ocotea corymbosa (Meisn.) Mez apresentaram os maiores VI. Juntas, totalizaram cerca de $55 \%$ do valor total da amostra. Podocarpus lambertii apresentou a maior freqüência, densidade e dominância relativas, ocorrendo em $78 \%$ dos pontos amostrados. Araucaria angustifolia e Cryptocarya aschersoniana apresentaram os maiores valores de dominância relativa, e Ocotea corymbosa e Mosiera prismatica os maiores valores de freqüência relativa (Tabela 4).

No grupo das espécies de maior porte também foram amostradas duas pteridófitas arborescentes: Alsophila setosa Kaulf. (Cyatheaceae) e Dicksonia sellowiana Hook. (Dicksoniaceae), mais freqüentemente observadas no estrato arbustivo da floresta. Foram amostradas e incluídas nas análises em função dos valores de perímetro dos indivíduos registrados em campo, compreendidos entre aqueles definidos como critério de inclusão para espécies de maior porte durante a amostragem.

Do total de espécies registradas no levantamento, 30 foram exclusivas do grupo definido como de menor porte, 20 exclusivas do grupo de maior porte e 47 foram comuns a ambos os grupos de amostragem (Tabela 2). 
Tabela 3. Parâmetros fitossociológicos das espécies amostradas na Floresta Ombrófila Mista Montana do Parque Municipal do Barigüi (Curitiba, PR) com PAP entre 10 e $29 \mathrm{~cm}$ - grupo de menor porte $(\mathrm{Ni}=$ número de indivíduos amostrados, $\mathrm{Np}=$ número de pontos de ocorrência da espécie, DA $=$ densidade absoluta, $\mathrm{DR}=$ densidade relativa $(\%)$, DoA $=$ dominância absoluta, DoR $=$ dominância relativa $(\%), \mathrm{FA}=$ freqüência absoluta, $\mathrm{FR}=$ freqüência relativa $(\%), \mathrm{VI}=$ valor de importância).

Table 3. Phytosociological parameters of the sampled species at the Mixed Ombrophilous Montane Forest at Barigüi Park (Curitiba, Paraná) with PBH between 10 and $29 \mathrm{~cm}$ - the lower size group $(\mathrm{Ni}=$ number of sampled individuals, $\mathrm{Np}=$ number of points of occurrence of species, $\mathrm{DA}=$ absolute density, $\mathrm{DR}=$ relative density $(\%), \mathrm{DoA}=$ absolute dominance, $\mathrm{DoR}=$ relative dominance $(\%), \mathrm{FA}=$ absolute frequency, $\mathrm{FR}=$ relative frequency $(\%), \mathrm{VI}=$ importance value).

\begin{tabular}{|c|c|c|c|c|c|c|c|c|c|}
\hline Espécies & $\mathbf{N i}$ & Np & DA & DR & DoA & DoR & FA & FR & VI \\
\hline Casearia decandra & 54 & 43 & 105,00 & 9,00 & 0,0023 & 8,62 & 28,67 & 8,40 & 26,02 \\
\hline Ocotea corymbosa & 52 & 41 & 101,11 & 8,67 & 0,0017 & 6,28 & 27,33 & 8,01 & 22,96 \\
\hline Ocotea nutans & 51 & 39 & 99,17 & 8,50 & 0,0018 & 6,38 & 26,00 & 7,62 & 22,49 \\
\hline Mosiera prismatica & 34 & 28 & 66,11 & 5,67 & 0,0031 & 7,42 & 18,67 & 5,47 & 18,56 \\
\hline Casearia sylvestris & 32 & 24 & 62,22 & 5,33 & 0,0026 & 5,98 & 16,00 & 4,69 & 16,00 \\
\hline Casearia obliqua & 28 & 23 & 54,45 & 4,67 & 0,0024 & 4,79 & 15,33 & 4,49 & 13,95 \\
\hline Cordiera concolor & 24 & 22 & 46,67 & 4,00 & 0,0028 & 4,83 & 14,67 & 4,30 & 13,13 \\
\hline Allophylus edulis & 20 & 16 & 38,89 & 3,33 & 0,0033 & 4,68 & 10,67 & 3,13 & 11,14 \\
\hline Myrceugenia miersiana & 20 & 17 & 38,89 & 3,33 & 0,0031 & 4,36 & 11,33 & 3,32 & 11,02 \\
\hline Psychotria vellosiana & 22 & 20 & 42,78 & 3,67 & 0,0021 & 3,21 & 13,33 & 3,91 & 10,78 \\
\hline Myrceugenia ovata & 25 & 18 & 48,61 & 4,17 & 0,0016 & 2,87 & 12,00 & 3,52 & 10,56 \\
\hline Eugenia uniflora & 20 & 18 & 38,89 & 3,33 & 0,0025 & 3,52 & 12,00 & 3,52 & 10,36 \\
\hline Solanum pseudoquina & 13 & 12 & 25,28 & 2,17 & 0,0030 & 2,73 & 8,00 & 2,34 & 7,24 \\
\hline Matayba elaeagnoides & 16 & 15 & 31,11 & 2,67 & 0,0014 & 1,62 & 10,00 & 2,93 & 7,22 \\
\hline Psychotria suterella & 13 & 11 & 25,28 & 2,17 & 0,0016 & 1,46 & 7,33 & 2,15 & 5,77 \\
\hline Podocarpus lambertii & 8 & 7 & 15,56 & 1,33 & 0,0048 & 2,74 & 4,67 & 1,37 & 5,44 \\
\hline Sebastiania brasiliensis & 9 & 6 & 17,50 & 1,50 & 0,0027 & 1,75 & 4,00 & 1,17 & 4,42 \\
\hline Ilex paraguariensis & 8 & 8 & 15,56 & 1,33 & 0,0019 & 1,07 & 5,33 & 1,56 & 3,97 \\
\hline Myrciaria tenella & 8 & 6 & 15,56 & 1,33 & 0,0022 & 1,27 & 4,00 & 1,17 & 3,78 \\
\hline Cupania vernalis & 8 & 8 & 15,56 & 1,33 & 0,0015 & 0,84 & 5,33 & 1,56 & 3,74 \\
\hline Xylosma ciliatifolium & 7 & 7 & 13,61 & 1,17 & 0,0024 & 1,17 & 4,67 & 1,37 & 3,70 \\
\hline Cabralea canjerana & 6 & 5 & 11,67 & 1,00 & 0,0023 & 0,96 & 3,33 & 0,98 & 2,94 \\
\hline Rapanea umbellata & 6 & 5 & 11,67 & 1,00 & 0,0022 & 0,94 & 3,33 & 0,98 & 2,92 \\
\hline Drimys brasiliensis & 6 & 6 & 11,67 & 1,00 & 0,0014 & 0,59 & 4,00 & 1,17 & 2,77 \\
\hline Myrcia hatschbachii & 5 & 5 & 9,72 & 0,83 & 0,0022 & 0,77 & 3,33 & 0,98 & 2,58 \\
\hline Sloanea monosperma & 4 & 4 & 7,78 & 0,67 & 0,0037 & 1,05 & 2,67 & 0,78 & 2,50 \\
\hline Myrceugenia myrcioides & 4 & 4 & 7,78 & 0,67 & 0,0034 & 0,98 & 2,67 & 0,78 & 2,42 \\
\hline Sebastiania commersoniana & 4 & 4 & 7,78 & 0,67 & 0,0032 & 0,90 & 2,67 & 0,78 & 2,35 \\
\hline Myrcia multiflora & 4 & 3 & 7,78 & 0,67 & 0,0038 & 1,08 & 2,00 & 0,59 & 2,33 \\
\hline Myrcia obtecta & 5 & 4 & 9,72 & 0,83 & 0,0016 & 0,56 & 2,67 & 0,78 & 2,17 \\
\hline Calyptranthes concinna & 4 & 4 & 7,78 & 0,67 & 0,0023 & 0,64 & 2,67 & 0,78 & 2,09 \\
\hline Rollinia emarginata & 4 & 4 & 7,78 & 0,67 & 0,0019 & 0,53 & 2,67 & 0,78 & 1,97 \\
\hline Mollinedia clavigera & 4 & 4 & 7,78 & 0,67 & 0,0015 & 0,44 & 2,67 & 0,78 & 1,89 \\
\hline Picramnia parvifolia & 3 & 3 & 5,83 & 0,50 & 0,0027 & 0,56 & 2,00 & 0,59 & 1,65 \\
\hline Celtis tala & 3 & 3 & 5,83 & 0,50 & 0,0023 & 0,49 & 2,00 & 0,59 & 1,58 \\
\hline Alsophila setosa & 2 & 2 & 3,89 & 0,33 & 0,0060 & 0,85 & 1,33 & 0,39 & 1,58 \\
\hline Eugenia platysema & 3 & 3 & 5,83 & 0,50 & 0,0016 & 0,34 & 2,00 & 0,59 & 1,43 \\
\hline Myrcia rostrata & 3 & 3 & 5,83 & 0,50 & 0,0016 & 0,33 & 2,00 & 0,59 & 1,42 \\
\hline Solanum sanctaecatharinae & 2 & 2 & 3,89 & 0,33 & 0,0048 & 0,68 & 1,33 & 0,39 & 1,40 \\
\hline Solanum swartzianum & 3 & 3 & 5,83 & 0,50 & 0,0015 & 0,31 & 2,00 & 0,59 & 1,40 \\
\hline Ilex brevicuspis & 3 & 3 & 5,83 & 0,50 & 0,0014 & 0,30 & 2,00 & 0,59 & 1,39 \\
\hline Myrcia cf. venulosa & 3 & 3 & 5,83 & 0,50 & 0,0013 & 0,27 & 2,00 & 0,59 & 1,36 \\
\hline Pimenta pseudocaryophyllus & 2 & 2 & 3,89 & 0,33 & 0,0035 & 0,50 & 1,33 & 0,39 & 1,22 \\
\hline Machaerium paraguariense & 2 & 2 & 3,89 & 0,33 & 0,0031 & 0,44 & 1,33 & 0,39 & 1,16 \\
\hline
\end{tabular}




\begin{tabular}{|c|c|c|c|c|c|c|c|c|c|}
\hline Espécies & $\mathbf{N i}$ & Np & DA & DR & DoA & DoR & FA & FR & VI \\
\hline Strychnos brasiliensis & 2 & 2 & 3,89 & 0,33 & 0,0031 & 0,44 & 1,33 & 0,39 & 1,16 \\
\hline Tetrapanax papyriferum & 2 & 2 & 3,89 & 0,33 & 0,0024 & 0,34 & 1,33 & 0,39 & 1,07 \\
\hline Ilex amara & 2 & 2 & 3,89 & 0,33 & 0,0019 & 0,27 & 1,33 & 0,39 & 1,00 \\
\hline Roupala brasiliensis & 2 & 2 & 3,89 & 0,33 & 0,0018 & 0,26 & 1,33 & 0,39 & 0,99 \\
\hline Campomanesia xanthocarpa & 2 & 2 & 3,89 & 0,33 & 0,0017 & 0,24 & 1,33 & 0,39 & 0,97 \\
\hline Guettarda uruguensis & 1 & 1 & 1,94 & 0,17 & 0,0080 & 0,57 & 0,67 & 0,20 & 0,93 \\
\hline Cinnamoтит sp. & 2 & 2 & 3,89 & 0,33 & 0,0014 & 0,20 & 1,33 & 0,39 & 0,92 \\
\hline Ocotea pulchella & 2 & 2 & 3,89 & 0,33 & 0,0013 & 0,19 & 1,33 & 0,39 & 0,91 \\
\hline Duranta vestita & 2 & 1 & 3,89 & 0,33 & 0,0026 & 0,37 & 0,67 & 0,20 & 0,90 \\
\hline Blepharocalyx salicifolius & 2 & 2 & 3,89 & 0,33 & 0,0010 & 0,14 & 1,33 & 0,39 & 0,86 \\
\hline Cedrela cf. odorata & 2 & 2 & 3,89 & 0,33 & 0,0008 & 0,11 & 1,33 & 0,39 & 0,84 \\
\hline Hovenia dulcis & 1 & 1 & 1,94 & 0,17 & 0,0066 & 0,47 & 0,67 & 0,20 & 0,83 \\
\hline Lonchocarpus subglaucescens & 1 & 1 & 1,94 & 0,17 & 0,0066 & 0,47 & 0,67 & 0,20 & 0,83 \\
\hline Zanthoxylum rhoifolium & 1 & 1 & 1,94 & 0,17 & 0,0066 & 0,47 & 0,67 & 0,20 & 0,83 \\
\hline Symplocos sp. & 1 & 1 & 1,94 & 0,17 & 0,0050 & 0,36 & 0,67 & 0,20 & 0,72 \\
\hline Symplocos tetrandra & 1 & 1 & 1,94 & 0,17 & 0,0050 & 0,36 & 0,67 & 0,20 & 0,72 \\
\hline Banara parviflora & 1 & 1 & 1,94 & 0,17 & 0,0050 & 0,36 & 0,67 & 0,20 & 0,72 \\
\hline Lithraea brasiliensis & 1 & 1 & 1,94 & 0,17 & 0,0042 & 0,30 & 0,67 & 0,20 & 0,66 \\
\hline Maytenus alaternoides & 1 & 1 & 1,94 & 0,17 & 0,0038 & 0,27 & 0,67 & 0,20 & 0,63 \\
\hline Lafoensia pacari & 1 & 1 & 1,94 & 0,17 & 0,0032 & 0,23 & 0,67 & 0,20 & 0,59 \\
\hline Maytenus ilicifolia & 1 & 1 & 1,94 & 0,17 & 0,0026 & 0,18 & 0,67 & 0,20 & 0,54 \\
\hline Capsicodendron dinisii & 1 & 1 & 1,94 & 0,17 & 0,0026 & 0,18 & 0,67 & 0,20 & 0,54 \\
\hline Allophylus guaraniticus & 1 & 1 & 1,94 & 0,17 & 0,0023 & 0,16 & 0,67 & 0,20 & 0,52 \\
\hline Dalbergia frutescens & 1 & 1 & 1,94 & 0,17 & 0,0020 & 0,14 & 0,67 & 0,20 & 0,51 \\
\hline Lamanonia ternata & 1 & 1 & 1,94 & 0,17 & 0,0018 & 0,13 & 0,67 & 0,20 & 0,49 \\
\hline Cinnamoтит aтоепит & 1 & 1 & 1,94 & 0,17 & 0,0018 & 0,13 & 0,67 & 0,20 & 0,49 \\
\hline Prunus sellowii & 1 & 1 & 1,94 & 0,17 & 0,0016 & 0,11 & 0,67 & 0,20 & 0,47 \\
\hline Ilex sp. & 1 & 1 & 1,94 & 0,17 & 0,0013 & 0,09 & 0,67 & 0,20 & 0,46 \\
\hline Machaerium brasiliense & 1 & 1 & 1,94 & 0,17 & 0,0011 & 0,08 & 0,67 & 0,20 & 0,44 \\
\hline Clethra scabra & 1 & 1 & 1,94 & 0,17 & 0,0010 & 0,07 & 0,67 & 0,20 & 0,43 \\
\hline Ilex theezans & 1 & 1 & 1,94 & 0,17 & 0,0008 & 0,06 & 0,67 & 0,20 & 0,42 \\
\hline Lantana brasiliensis & 1 & 1 & 1,94 & 0,17 & 0,0008 & 0,06 & 0,67 & 0,20 & 0,42 \\
\hline Baccharis brachylaenoides & 1 & 1 & 1,94 & 0,17 & 0,0008 & 0,06 & 0,67 & 0,20 & 0,42 \\
\hline
\end{tabular}

Tabela 4. Parâmetros fitossociológicos das espécies amostradas na Floresta Ombrófila Mista Montana do Parque Municipal do Barigüi (Curitiba, PR), com PAP igual ou maior do que $30 \mathrm{~cm}$ - grupo de maior porte $(\mathrm{Ni}=$ número de indivíduos amostrados, $\mathrm{Np}=$ número de pontos de ocorrência da espécie, $\mathrm{DA}=$ densidade absoluta, $\mathrm{DR}=$ densidade relativa $(\%)$, DoA = dominância absoluta, $\mathrm{DoR}=$ dominância relativa $(\%), \mathrm{FA}=$ freqüência absoluta, $\mathrm{FR}=$ freqüência relativa $(\%), \mathrm{VI}=$ valor de importância).

Table 4. Phytosociological parameters of the sampled species at the Mixed Ombrophilous Montane Forest at Barigüi Park (Curitiba, Paraná) with PBH equal or above $30 \mathrm{~cm}-$ the higher size group $(\mathrm{Ni}=$ number of sampled individuals, $\mathrm{Np}=$ number of points of occurrence of species, $\mathrm{DA}=$ absolute density, $\mathrm{DR}=$ relative density $(\%), \mathrm{DoA}=$ absolute dominance, $\mathrm{DoR}=$ relative dominance $(\%), \mathrm{FA}=$ absolute frequency, $\mathrm{FR}=$ relative frequency $(\%), \mathrm{VI}=$ importance value).

\begin{tabular}{llllllllll}
\hline Espécies & Ni & Np & DA & DR & DoA & DoR & FA & FR & VI \\
\hline Podocarpus lambertii & 254 & 117 & 271,59 & 42,33 & 0,0537 & 41,02 & 78,00 & 27,86 & 111,21 \\
Mosiera prismatica & 51 & 37 & 54,53 & 8,50 & 0,0366 & 5,61 & 24,67 & 8,81 & 22,92 \\
Araucaria angustifolia & 16 & 13 & 17,11 & 2,67 & 0,2477 & 11,92 & 8,67 & 3,10 & 17,68 \\
Ocotea corymbosa & 28 & 23 & 29,94 & 4,67 & 0,0608 & 5,12 & 15,33 & 5,48 & 15,26 \\
Cryptocarya aschersoniana & 16 & 14 & 17,11 & 2,67 & 0,1762 & 8,48 & 9,33 & 3,33 & 14,48 \\
Casearia obliqua & 23 & 20 & 24,59 & 3,83 & 0,0366 & 2,53 & 13,33 & 4,76 & 11,13 \\
Eugenia uniflora & 21 & 17 & 22,45 & 3,50 & 0,0196 & 1,24 & 11,33 & 4,05 & 8,79 \\
Vernonia discolor & 13 & 10 & 13,90 & 2,17 & 0,0631 & 2,47 & 6,67 & 2,38 & 7,01 \\
\hline
\end{tabular}




\begin{tabular}{|c|c|c|c|c|c|c|c|c|c|}
\hline Espécies & $\mathbf{N i}$ & $\mathbf{N p}$ & DA & DR & DoA & DoR & FA & FR & VI \\
\hline Ocotea nutans & 9 & 8 & 9,62 & 1,50 & 0,1079 & 2,92 & 5,33 & 1,90 & 6,33 \\
\hline Capsicodendron dinisii & 11 & 10 & 11,76 & 1,83 & 0,0392 & 1,30 & 6,67 & 2,38 & 5,51 \\
\hline Alsophila setosa & 13 & 12 & 13,90 & 2,17 & 0,0093 & 0,36 & 8,00 & 2,86 & 5,39 \\
\hline Casearia sylvestris & 11 & 10 & 11,76 & 1,83 & 0,0148 & 0,49 & 6,67 & 2,38 & 4,71 \\
\hline Prunus sellowii & 10 & 8 & 10,69 & 1,67 & 0,0313 & 0,94 & 5,33 & 1,90 & 4,51 \\
\hline Myrcia multiflora & 8 & 7 & 8,55 & 1,33 & 0,0389 & 0,94 & 4,67 & 1,67 & 3,94 \\
\hline Sebastiania commersoniana & 7 & 6 & 7,48 & 1,17 & 0,0311 & 0,65 & 4,00 & 1,43 & 3,25 \\
\hline Drimys brasiliensis & 6 & 6 & 6,42 & 1,00 & 0,0374 & 0,67 & 4,00 & 1,43 & 3,10 \\
\hline Matayba elaeagnoides & 3 & 3 & 3,21 & 0,50 & 0,1686 & 1,52 & 2,00 & 0,71 & 2,74 \\
\hline Lamanonia ternata & 4 & 4 & 4,28 & 0,67 & 0,0681 & 0,82 & 2,67 & 0,95 & 2,44 \\
\hline Myrcia sp. & 3 & 3 & 3,21 & 0,50 & 0,0946 & 0,85 & 2,00 & 0,71 & 2,07 \\
\hline Lafoensia pacari & 2 & 2 & 2,14 & 0,33 & 0,1945 & 1,17 & 1,33 & 0,48 & 1,98 \\
\hline Ilex brevicuspis & 4 & 4 & 4,28 & 0,67 & 0,0204 & 0,25 & 2,67 & 0,95 & 1,86 \\
\hline Ocotea puberula & 3 & 3 & 3,21 & 0,50 & 0,0701 & 0,63 & 2,00 & 0,71 & 1,85 \\
\hline Allophylus edulis & 4 & 4 & 4,28 & 0,67 & 0,0185 & 0,22 & 2,67 & 0,95 & 1,84 \\
\hline Casearia decandra & 4 & 4 & 4,28 & 0,67 & 0,0181 & 0,22 & 2,67 & 0,95 & 1,84 \\
\hline Clethra scabra & 3 & 3 & 3,21 & 0,50 & 0,0639 & 0,58 & 2,00 & 0,71 & 1,79 \\
\hline Myrcia hatschbachii & 3 & 3 & 3,21 & 0,50 & 0,0516 & 0,47 & 2,00 & 0,71 & 1,68 \\
\hline Jacaranda puberula & 3 & 3 & 3,21 & 0,50 & 0,0440 & 0,40 & 2,00 & 0,71 & 1,61 \\
\hline Symplocos tetrandra & 4 & 3 & 4,28 & 0,67 & 0,0184 & 0,22 & 2,00 & 0,71 & 1,60 \\
\hline Zanthoxylum kleinii & 3 & 3 & 3,21 & 0,50 & 0,0365 & 0,33 & 2,00 & 0,71 & 1,54 \\
\hline Myrceugenia myrcioides & 3 & 3 & 3,21 & 0,50 & 0,0300 & 0,27 & 2,00 & 0,71 & 1,49 \\
\hline Rapanea umbellata & 3 & 3 & 3,21 & 0,50 & 0,0163 & 0,15 & 2,00 & 0,71 & 1,36 \\
\hline Myrceugenia miersiana & 3 & 3 & 3,21 & 0,50 & 0,0153 & 0,14 & 2,00 & 0,71 & 1,35 \\
\hline Psychotria vellosiana & 3 & 3 & 3,21 & 0,50 & 0,0140 & 0,13 & 2,00 & 0,71 & 1,34 \\
\hline Ilex dumosa & 2 & 2 & 2,14 & 0,33 & 0,0853 & 0,51 & 1,33 & 0,48 & 1,32 \\
\hline Campomanesia xanthocarpa & 2 & 2 & 2,14 & 0,33 & 0,0523 & 0,31 & 1,33 & 0,48 & 1,12 \\
\hline Blepharocalyx salicifolius & 2 & 2 & 2,14 & 0,33 & 0,0491 & 0,30 & 1,33 & 0,48 & 1,11 \\
\hline Lithraea brasiliensis & 2 & 2 & 2,14 & 0,33 & 0,0478 & 0,29 & 1,33 & 0,48 & 1,10 \\
\hline Maytenus evonymoides & 1 & 1 & 1,07 & 0,17 & 0,2035 & 0,61 & 0,67 & 0,24 & 1,02 \\
\hline Lonchocarpus subglaucescens & 2 & 2 & 2,14 & 0,33 & 0,0328 & 0,20 & 1,33 & 0,48 & 1,01 \\
\hline Rapanea lancifolia & 2 & 2 & 2,14 & 0,33 & 0,0245 & 0,15 & 1,33 & 0,48 & 0,96 \\
\hline Myrcia rostrata & 2 & 2 & 2,14 & 0,33 & 0,0187 & 0,11 & 1,33 & 0,48 & 0,92 \\
\hline Celtis tala & 2 & 2 & 2,14 & 0,33 & 0,0184 & 0,11 & 1,33 & 0,48 & 0,92 \\
\hline Solanum swartzianum & 2 & 2 & 2,14 & 0,33 & 0,0184 & 0,11 & 1,33 & 0,48 & 0,92 \\
\hline Zanthoxylum rhoifolium & 2 & 2 & 2,14 & 0,33 & 0,0180 & 0,11 & 1,33 & 0,48 & 0,92 \\
\hline Machaerium brasiliense & 2 & 2 & 2,14 & 0,33 & 0,0158 & 0,09 & 1,33 & 0,48 & 0,90 \\
\hline Solanum sanctaecatharinae & 2 & 2 & 2,14 & 0,33 & 0,0133 & 0,08 & 1,33 & 0,48 & 0,89 \\
\hline Allophylus guaraniticus & 2 & 2 & 2,14 & 0,33 & 0,0119 & 0,07 & 1,33 & 0,48 & 0,88 \\
\hline Solanum pseudoquina & 2 & 2 & 2,14 & 0,33 & 0,0101 & 0,06 & 1,33 & 0,48 & 0,87 \\
\hline Syagrus romanzoffiana & 1 & 1 & 1,07 & 0,17 & 0,1069 & 0,32 & 0,67 & 0,24 & 0,73 \\
\hline Schinus terebinthifolius & 1 & 1 & 1,07 & 0,17 & 0,0716 & 0,22 & 0,67 & 0,24 & 0,62 \\
\hline Erythroxylum deciduum & 1 & 1 & 1,07 & 0,17 & 0,0642 & 0,19 & 0,67 & 0,24 & 0,60 \\
\hline Persea major & 1 & 1 & 1,07 & 0,17 & 0,0590 & 0,18 & 0,67 & 0,24 & 0,58 \\
\hline Styrax martii & 1 & 1 & 1,07 & 0,17 & 0,0423 & 0,13 & 0,67 & 0,24 & 0,53 \\
\hline Machaerium stipitatum & 1 & 1 & 1,07 & 0,17 & 0,0423 & 0,13 & 0,67 & 0,24 & 0,53 \\
\hline Xylosma ciliatifolium & 1 & 1 & 1,07 & 0,17 & 0,0356 & 0,11 & 0,67 & 0,24 & 0,51 \\
\hline Ilex theezans & 1 & 1 & 1,07 & 0,17 & 0,0346 & 0,10 & 0,67 & 0,24 & 0,51 \\
\hline Cinnamomum amoenum & 1 & 1 & 1,07 & 0,17 & 0,0224 & 0,07 & 0,67 & 0,24 & 0,47 \\
\hline Maytenus aquifolium & 1 & 1 & 1,07 & 0,17 & 0,0224 & 0,07 & 0,67 & 0,24 & 0,47 \\
\hline Dicksonia sellowiana & 1 & 1 & 1,07 & 0,17 & 0,0216 & 0,07 & 0,67 & 0,24 & 0,47 \\
\hline Sebastiania brasiliensis & 1 & 1 & 1,07 & 0,17 & 0,0177 & 0,05 & 0,67 & 0,24 & 0,46 \\
\hline Lonchocarpus cultratus & 1 & 1 & 1,07 & 0,17 & 0,0161 & 0,05 & 0,67 & 0,24 & 0,45 \\
\hline Solanum granuloso-leprosum & 1 & 1 & 1,07 & 0,17 & 0,0154 & 0,05 & 0,67 & 0,24 & 0,45 \\
\hline Myrcia obtecta & 1 & 1 & 1,07 & 0,17 & 0,0109 & 0,03 & 0,67 & 0,24 & 0,44 \\
\hline Pimenta pseudocaryophyllus & 1 & 1 & 1,07 & 0,17 & 0,0104 & 0,03 & 0,67 & 0,24 & 0,44 \\
\hline Ocotea pulchella & 1 & 1 & 1,07 & 0,17 & 0,0104 & 0,03 & 0,67 & 0,24 & 0,44 \\
\hline Cabralea canjerana & 1 & 1 & 1,07 & 0,17 & 0,0082 & 0,02 & 0,67 & 0,24 & 0,43 \\
\hline Duranta vestita & 1 & 1 & 1,07 & 0,17 & 0,0077 & 0,02 & 0,67 & 0,24 & 0,43 \\
\hline
\end{tabular}




\section{DISCUSSÃO}

Com os resultados obtidos durante a amostragem, foi possível caracterizar os estratos inferior e superior da floresta. Os intervalos de PAP estabelecidos para a avaliação das espécies, supostamente de menor e maior porte, foram satisfatórios. Com exceção a duas pteridófitas, as demais espécies amostradas dentro de cada um dos grupos caracterizaram-se, pela altura, como constituintes do componente inferior e superior da floresta, respectivamente.

As duas pteridófitas mencionadas apresentam, naturalmente, crescimento típico arborescente. Apesar de terem sido incluídas durante a amostragem no grupo das espécies de maior porte, em função do critério de inclusão estabelecido, tiveram registro de valores de altura próximos ao valor de altura média constatado para as espécies de menor porte, indicando com isso presença efetiva no estrato inferior da floresta. O crescimento em espessura dessas espécies resulta no desenvolvimento de indivíduos com elevados valores de PAP, mas não necessariamente com elevados valores de altura, conforme hipótese considerada no início do trabalho. Por apresentar esse tipo de desenvolvimento, foram amostradas entre os indivíduos de maior porte.

Araucaria angustifolia e Cryptocarya aschersoniana destacaram-se no grupo das espécies de maior porte com elevados VI, principalmente devido aos valores de dominância, que refletem o grande porte dos indivíduos. Essas espécies não foram amostradas no grupo de menor porte, o que evidencia uma baixa regeneração natural dentro da comunidade. A coleta de sementes, a predação por animais, especialmente com relação a Araucaria angustifolia, e os processos naturais de sucessão estão entre os principais fatores que justificam a ausência da regeneração dessas espécies na floresta.

Ao contrário dessas, Podocarpus lambertii, Ocotea porosa e Mosiera prismatica destacaram-se no levantamento nos dois grupos de amostragem e estão entre as mais importantes.

Podocarpus lambertii, em especial, além de ter apresentado o maior VI do grupo de maior porte, foi a espécie com maior número de indivíduos amostrados no levantamento total. Sua presença é muito importante na estrutura da floresta do Parque Barigüi, que é, em parte, semelhante às comunidades florestais observadas por Backes (1983). Esse autor, em estudos realizados em diferentes florestas, cita que Podocarpus lambertii pode apresentar, em uma mesma área com FOM, uma sucessão de indivíduos com diferentes idades, desde aqueles em processo de regeneração natural até os que se encontram ocupando as porções mais altas da comunidade.

Já em Jarenkow e Baptista (1987) e Negrelle e Silva (1992), a presença de Podocarpus lambertii como principal elemento da organização estrutural da floresta não foi observada. Nos estudos efetuados, sequer citaram a presença da espécie na composição de FOM, provavelmente substituída no sistema por outras espécies de estágios sucessionais mais desenvolvidos.

Myrtaceae, Lauraceae e Flacourtiaceae, famílias que se destacaram em número de espécies e nos demais parâmetros neste trabalho, também foram citadas como as mais ricas em outros trabalhos fitossociológicos realizados no Sul do país, como em Oliveira e Rota (1980), Jarenkow e Baptista (1987), Silva e Marconi (1990), Negrelle e Silva (1992), Silva et al. (1997) e Neto et al. (2002). Representam famílias de grande importância florística e estrutural em áreas de FOM. No Parque Barigüi, em especial, foram representadas principalmente por Mosiera prismatica (Myrtaceae), Ocotea corymbosa, Ocotea nutans, Cryptocarya aschersoniana (Lauraceae), Casearia decandra, Casearia sylvestris e Casearia obliqua (Flacourtiaceae).

Dentre as espécies de Flacourtiaceae, comuns no interior da floresta, destacou-se Casearia decandra com maior VI no grupo de menor porte. Também foi citada por Klein (1979) como uma das principais espécies presentes na constituição do estrato inferior de áreas de FOM localizadas no vale do Itajaí, SC. Nessa localidade, Casearia decandra, juntamente com outras do gênero Ilex (Aquifoliaceae), foi citada como uma das mais características do interior florestal e que apresenta grande dispersão ao longo dos "pinhais" (FOM). No Parque Barigüi, da mesma forma como observado em Itajaí, Casearia decandra destacou-se em importância na constituição do interior da floresta, juntamente com espécies de Lauraceae, Myrtaceae e Rubiaceae. As espécies de Aquifoliaceae foram registradas, mas não tiveram a mesma importância em termos estruturais.

Importante destacar a presença de Araucaria angustifolia na constituição da estrutura da floresta. Por ocupar o estrato superior, destacou-se no fragmento com indivíduos emergentes, os quais, ocupando essa posição na floresta, imprimem característica fitofisionômica típica e exclusiva de áreas com FOM. A 
regeneração natural dessa espécie no fragmento do Parque Barigüi, no entanto, tem limitações, principalmente devido à coleta de sementes por visitantes e predadores naturais e ao isolamento da floresta por áreas urbanizadas, não tendo, neste caso, condições de expandir sua área de ocupação. Sem possibilidade de regeneração, possivelmente os indivíduos adultos da floresta, em declínio natural devido ao processo de senescência, poderão deixar de constituir parte do sistema e serão substituídos por outras espécies do processo de sucessão, com conseqüente modificação da fisionomia florestal.

Com relação à diversidade da floresta, uma comparação entre os dois grupos de amostragem indicou que essa foi maior no estrato inferior do que no superior. O grupo de menor porte foi representado por um número maior de espécies, resultante da associação daquelas com ocorrência exclusiva no estrato inferior da floresta e de outras representadas por indivíduos do grupo das espécies de maior porte em regeneração natural.

Neto et al. (2002), em levantamento fitossociológico realizado em um fragmento com FOM em Curitiba, obtiveram valores de diversidade, número de espécies amostradas, famílias mais ricas, espécies mais importantes e altura média da floresta próximos aos valores registrados para o estrato inferior do Parque Barigüi. As semelhanças, quanto aos resultados obtidos, provavelmente estão relacionadas ao fato de as áreas encontrarem-se relativamente próximas, quanto à localização, e em condições físicas e climáticas similares. Importante destacar que, ao serem considerados os dois grupos de espécies estudados na floresta do Parque Barigüi, ou seja, o componente do estrato inferior e do superior, a riqueza específica da floresta passa a somar um número muito maior de espécies e, por isso, comparativamente aos resultados de Neto et al. (2002), uma diversidade maior. A isso atribui-se principalmente a grande heterogeneidade ambiental e o grau de conservação da vegetação.

\section{AGRADECIMENTOS}

Ao CNPq (bolsas de iniciação científica), à Secretaria Estadual de Meio Ambiente de Curitiba (autorização para a realização do trabalho), ao Departamento de Botânica da Universidade Federal do Paraná (utilização dos laboratórios) e aos amigos e familiares que colaboraram, principalmente durante as atividades de campo.

\section{REFERÊNCIAS}

ABE, L. A.; BARDDAL, M. L.; BERNARDI, D. Mapeamento e caracterização da cobertura vegetal do Parque Barigüi, Curitiba, Paraná. In: PESQUISA FLORESTAL ONLINE, 2000, Curitiba Anais...Curitiba: UFPR, 2000. p.129.

BACKES, A. Dinâmica do pinheiro-brasileiro. Iheringia, Série Botânica, Porto Alegre, n. 30, p. 49-84, 1983.

BRITEZ, R. M.; SILVA, S. M.; SOUZA, W. S.; MOTTA, J. T. W. Levantamento florístico em Floresta Ombrófila Mista, São Mateus do Sul, Paraná, Brasil. Arquivos de Biologia e Tecnologia, Curitiba, n. 38, p. 1147-1161, 1995.

CERVI, A. C.; ACRA, L. A.; RODRIGUES, L.; GABRIEL, M. M.; LOPES, M. Contribuição ao conhecimento das plantas herbáceas de uma floresta de Araucária do primeiro planalto paranaense. Ínsula, Florianópolis, n. 18, p. 83-98, 1988.

CERVI, A. C.; ACRA, L. A.; RODRIGUES, L.; TRAIN, S.; IVANCHECHEN, S. L.; MOREIRA, A. L. O. R. Contribuição ao conhecimento das pteridófitas de uma mata de Araucária, Curitiba, Paraná, Brasil. Acta Biológica Paranaense, Curitiba, n. 16, p. 77-85, 1987 a.

CERVI, A. C.; PACIORNIK, E. F.; VIEIRA, R. F.; MARQUES, L. C. Espécies vegetais de um remanescente de floresta de Araucária (Curitiba, BR): Estudo preliminar I. Acta Biológica Paranaense, Curitiba, n. 18, p. 73-114, 1989.

CERVI, A. C.; SCHIMMELPFENG, L. C. T.; PASSOS, M. Levantamento do estrato arbóreo do Capão da Educação Física da UFPR, Curitiba, Paraná, Brasil. Estudos de Biologia, Curitiba, n. 17, p. 49-61, $1987 b$.

FLORESTA, Curitiba, PR, v. 36, n. 2, mai./ago. 2006. 
CESTARO, L. A.; WAECHTER, J. L.; BAPTISTA, L. R. M. Fitossociologia do estrato herbáceo da mata de Araucária da Estação Ecológica de Aracuri, Esmeralda, RS. Hoehnea, São Paulo, n. 13, p. 59-72, 1986.

DITTRICH, V. A. O.; KOZERA, C.; MENEZES-SILVA, S. Levantamento florístico dos epífitos vasculares do Parque Barigüi, Curitiba, Paraná, Brasil. Iheringia, Série Botânica, Porto Alegre, n. 52, p. 11-22, 1999.

DOMBROWSKI, L. T. D.; KUNIYOSHI, Y. S. A vegetação do “Capão da Imbuia”. Araucariana. Série Botânica, Curitiba, n. 1, p. 1-18, 1967.

GALVÃO, F.; KUNIYOSHI, Y. S.; RODERJAN, C. V. Levantamento fitossociológico das principais associações arbóreas da Floresta Nacional de Irati - PR. Floresta, Curitiba, n. 19, p. 30-49, 1989.

GRAF, A. B. Tropica color cyclopedia of exotic plants and trees. 4.ed. USA: Roehrs Company, 1992.

HATSCHBACH, G.; MOREIRA FILHO, H. Catálogo florístico do Parque de Vila Velha (Estado do Paraná - Brasil). Boletim da Universidade Federal do Paraná, Curitiba, n. 28, p. 1-49, 1972.

IAPAR. Cartas climáticas básicas do Estado do Paraná. Londrina, 1978.

IBGE. Manual técnico da vegetação brasileira. Rio de Janeiro, 1992.

IPNI - THE INTERNATIONAL PLANT NAMES INDEX. The international plant names index database. Disponível em: <http://www.ipni.org/index.html>. Acesso em abr. 2006.

JARENKOW, J. A.; BAPTISTA, L. R. M. Composição florística e estrutura da mata com Araucaria angustifolia na Estação Ecológica de Aracuri, Esmeralda, Rio Grande do Sul. Napaea: Revista de Botanica, Porto Alegre, n. 3, p. 9-18, 1987.

KLEIN, R. M. O aspecto dinâmico do pinheiro brasileiro. Sellowia, Itajaí, n. 12, p. 17-44, 1960.

KLEIN, R. M. Observações e considerações sobre a vegetação do planalto nordeste catarinense. Sellowia, Itajaí, n. 15, p. 39-57, 1963.

KLEIN, R. M. Ecologia da flora e vegetação do Vale do Itajaí. Sellowia, Itajaí, n. 31, p. 11-164, 1979.

LEITE, P. F.; KLEIN, R. M. Vegetação. In: Geografia do Brasil - Região Sul. Rio de Janeiro: IBGE, 1990. v. 2, p. 113-150.

LONGHI, S. J. A estrutura de uma floresta natural de Araucaria angustifolia (Bertol.) O. Kuntze no sul do Brasil. 198f. Dissertação (Mestrado em Engenharia Florestal) - Setor de Ciências Agrárias, Universidade Federal do Paraná, Curitiba. 1980.

LORENZI, H.; SOUZA, H. M.; TORRES, M. A. V.; BACHER, L. B. Árvores exóticas no Brasil: madeireiras, ornamentais e aromáticas. Nova Odessa, : Instituto Plantarum, 2003.

MACHADO, S. A.; FIGUEIREDO, D. J.; HOSOKAWA, R. T. Composição estrutural e quantitativa de uma floresta secundária do norte catarinense. Revista do Instituto Florestal, São Paulo n. 4, p. 513-518, 1992.

MARTAU, L.; AGUiAR, L. W.; SOARES, Z. F.; BUENO, O. L. Estudo florístico do Parque dos Pinheiros e Centro de Lazer e Recreação Santa Rita, município de Farroupilha, RS, Brasil. Iheringia, Série Botânica, Porto Alegre, n. 28, p. 17-43, 1981.

MARTINS, F. R. Estrutura de uma floresta mesófila. 2.ed. Campinas, SP: Editora da UNICAMP, 1993.

MISSOURI BOTANICAL GARDEN. Missouri Botanical Garden's VAST (Vascular Tropicos) nomenclatural database and associated authority files. Disponível em: < http://mobot.org/ W3T/Search/vast.html> Acesso em abr. 2006. 
NEGRELLE, R. R. B.; SILVA, F. C. Fitossociologia de um trecho de floresta com Araucaria angustifolia (Bertol.) O. Kuntze no município de Caçador, Santa Catarina. Boletim de Pesquisas Florestais,Colombo, n. 24/25, p. 37-54, 1992.

NEGRELLE, R. R. B.; LEUCHTENBERGER, R. Composição e estrutura do componente arbóreo de um remanescente de Floresta Ombrófila Mista. Floresta, Curitiba, n. 31, p. 42-51, 2001.

OLIVEIRA, Y. M. M.; ROTTA, E. Levantamento da estrutura horizontal de uma mata de Araucária do primeiro planalto paranaense. Boletim de Pesquisas Florestais, Colombo, n. 4, p. 1-46, 1980.

RONDON NETO, R. M.; KOZERA, C.; ANDRADE, R. R.; CECY, A. T.; HUMMES, A. P.; FRITZSONS, E.; CALDEIRA, M. V. W.; MACIEL, M. N. M.; SOUZA, M. K. F. Caracterização florística e estrutural de um fragmento de Floresta Ombrófila Mista em Curitiba, PR, BR. Floresta, Curitiba, n. 32, p. 3-16, 2002.

ROSEIRA, D. S. Composição florística e estrutura fitossociológica do Bosque com Araucaria angustifolia (Bert.) O. Ktze no Parque Estadual João Paulo II, Curitiba, Paraná. 107f. Dissertação (Mestrado ) - Setor de Ciências Biológicas, Universidade Federal do Paraná, Curitiba. 1990.

SENNA, R. M.; WAECHTER, J. L. Pteridófitas de uma floresta com araucária. I. Formas biológicas e padrões de distribuição geográfica. Iheringia, Série Botânica, Porto Alegre, n. 48, p. 41-58, 1997.

SHEPHERD, G. J. Fitopac 1: manual do usuário. Campinas: Universidade Estadual de Campinas, 1995.

SILVA, F. C.; MARCONI, L. P. Fitossociologia de uma floresta com Araucária em Colombo - PR. Boletim de Pesquisas Florestais, Colombo, n. 20, p. 23-38, 1990.

SILVA, J. A.; SALOMÃO, A. N.; NETTO, D. A. M. Natural regeneration under Araucaria angustifolia (Bert.) O. Ktze. forest in the genetic reserve of Caçador - SC. Revista Árvore, Viçosa, MG, n. 22, p. 143153, 1998.

SILVA, J. A.; SALOMÃO, A. N.; GRIPP, A.; LEITE, E. J. Phytososiological survey in Brazil forest genetic reserve of Caçador. Plant Ecology, Dordrecht, n. 133, p. 1-11, 1997.

VELOSO, H. P.; RANGEL-FILHO, A. L. R.; LIMA, I. C. A. Classificação da vegetação brasileira, adaptada a um sistema universal. Rio de Janeiro: IBGE, 1991. 